\title{
Long cycles in subgraphs of (pseudo)random directed graphs
}

\author{
Ido Ben-Eliezer* Michael Krivelevich ${ }^{\dagger} \quad$ Benny Sudakov ${ }^{\ddagger}$
}

October 23, 2018

\begin{abstract}
We study the resilience of random and pseudorandom directed graphs with respect to the property of having long directed cycles. For every $0<\gamma<1 / 2$ we find a constant $c=c(\gamma)$ such that the following holds. Let $G=(V, E)$ be a (pseudo)random directed graph on $n$ vertices, and let $G^{\prime}$ be a subgraph of $G$ with $(1 / 2+\gamma)|E|$ edges. Then $G^{\prime}$ contains a directed cycle of length at least $(c-o(1)) n$. Moreover, there is a subgraph $G^{\prime \prime}$ of $G$ with $(1 / 2+\gamma-o(1))|E|$ edges that does not contain a cycle of length at least cn.
\end{abstract}

\section{Introduction}

Given a property $\mathcal{P}$, a typical problem in extremal graph theory can be stated as follows. Given a number of vertices $n$, what is the minimal (or maximal) number $f_{\mathcal{P}}(n)$ such that any graph on $n$ vertices with $f(n)$ edges possesses $\mathcal{P}$ ? Many examples of such problems and results can be found, e.g., in [7].

Usually, the property $\mathcal{P}$ we consider in extremal problems is either monotone increasing or monotone decreasing. A property $\mathcal{P}$ is monotone increasing (respectively, decreasing) if it preserved under edge addition (respectively, deletion).

The resilience of a graph $G$ with respect to a property $\mathcal{P}$ measures how far the graph is from any graph $H$ that does not have $\mathcal{P}$. In particular, the study of resilience usually focuses on monotone properties, and the following two types of problems are studied.

*School of Computer Science, Raymond and Beverly Sackler Faculy of Exact Sciences, Tel Aviv University, Tel Aviv 69978, Israel, e-mail: idobene@post.tau.ac.il. Research supported in part by an ERC advanced grant.

${ }^{\dagger}$ School of Mathematical Sciences, Raymond and Beverly Sackler Faculty of Exact Sciences, Tel Aviv University, Tel Aviv 69978, Israel, e-mail: krivelev@post.tau.ac.il. Research supported in part by USA-Israel BSF grant 2006322 and by grant 1063/08 from the Israel Science Foundation.

‡Department of Mathematics, UCLA, Los Angeles, CA 90095. Email: bsudakov@math.ucla.edu. Research supported in part by NSF CAREER award DMS-0812005 and by a USA-Israeli BSF grant. 
Global Resilience. Given a monotone increasing property $\mathcal{P}$, the global resilience of $G$ respect to $\mathcal{P}$ is the maximal integer $R$ such that for every subset $E_{0} \subseteq E(G)$ of $\left|E_{0}\right|=R$ edges, the graph $G-E_{0}$ still possesses $\mathcal{P}$. For the case of a monotone decreasing property $\mathcal{P}$, the global resilience is defined as the maximum number $R$ such that the addition of any subset of $R$ edges to $G$ still results in a graph $G^{\prime} \in \mathcal{P}$.

One can also define the notion of local resilience of a graph with respect to, say, a monotone increasing property $\mathcal{P}$ as the maximum number $r$ such that for any subgraph $H \subseteq G$ of maximum degree $r$, the graph $G-H$ is still in $\mathcal{P}$. Since in this paper we will be concerned with properties related to global resilience, we will not dwell on the notion of local resilience anymore.

The explicit study of this notion was initiated by Sudakov and Vu [17, but in a sense many well known theorems in extremal graph theory can be stated using this terminology. For example, given a fixed graph $H$, the Turán number of $H$, denoted by $e x(H, n)$, is the minimum number $m$ such that any graph on $n$ vertices with $m$ edges contains a copy of $H$. Clearly, the study of Turán numbers is equivalent to the study of the global resilience of the complete graph $K_{n}$ with respect to the property of having a copy of $H$.

Woodall [19] gave tight bounds for the number of edges in an undirected graph that guarantees the existence of a cycle of length at least $\ell$. In our terminology, he gave tight bounds on the global resilience of $K_{n}$ with respect to the property of having a cycle of length at least $\ell$. We will discuss Woodall's result later and will also use his result in our work. Lewin [16] studied the analogous problem for directed graphs, and he gave tight bounds on the number of edges required for having a directed cycle of length at least $\ell$. Many extremal results regarding the existence of cycles in directed graphs can be found, e.g., in [6].

Recently, there has been an extensive line of works that studying the resilience of graphs with respect to different properties. Dellamonica et al. [10] studied the local and global resilience of long cycles in pseudorandom undirected graphs. Krivelevich et al. [15] studied the resilience with respect to pancyclicity (having cycle of every possible length). Ben-Shimon et al. [5] studied the resilience of several graph properties in random regular graphs. Alon and Sudakov [1] studied the resilience of the chromatic number in random graphs. Huang et al. 13] studied the resilience with respect to having a spanning graph $H$ as a subgraph, answering a question of Böttcher et al. [8]. Balogh et al. [3] studied the resilience of random and pseudorandom graphs with respect to containing a copy of a given nearly spanning tree of bounded maximum degree.

Here we study the resilience of pseudorandom (and hence, of random) directed graphs with respect to the property of having a long directed cycle (namely, a simple directed cycle that covers a constant fraction of the vertices). We prove asymptotically tight bounds, and thus provide the asymptotic value of the resilience of every graph with respect to this property, assuming it has some predefined pseudorandomness property. Our proof applies a variant of the celebrated Szemerédi's regularity lemma for sparse directed graphs, and a short and simple technique for finding a long directed path in pseudorandom directed graphs. Using these techniques we can reduce our problem to the case of undirected graphs, where by applying techniques of [10] we can give tight bounds. 


\subsection{The models}

We consider here directed graphs on $n$ vertices, where antiparallel edges are allowed. We say that a graph $D=(V, E)$ has density $p$ if $|E|=p n^{2}$.

Let $D(n, p)$ be the following natural distribution of random directed graphs. Every graph in the support of $D(n, p)$ contains $n$ vertices, and for every two distinct vertices $x, y$, there is an edge from $x$ to $y$ with probability $p$, and independently there is an edge from $y$ to $x$ with probability $p$. Clearly, the expected number of edges is $2 p\left(\begin{array}{l}n \\ 2\end{array}\right)$.

Once we define our random digraph model, it is usually desirable to define a pseudorandom analog. That is, we would like to define a property such that graphs with this property have many of the 'nice' properties of random graphs. Roughly speaking, we say that a directed graph is pseudorandom if the number of edges between every two large enough sets is close to the expected number of edges in a random directed graph with the same density. More formally, we say that a directed graph $G$ is $(p, r)$-pseudorandom if it has edge density $p$ and for every two disjoint sets $A, B \subseteq V(G),|A|=|B|$, the number of edges from $A$ to $B$, denoted by $e_{G}(A, B)$, satisfies

$$
\left|e_{G}(A, B)-p\right| A|| B|| \leq r|A| \sqrt{p n} .
$$

This is (up to normalization) a directed variant of the well known notion of jumbled graphs, that was introduced By Thomason [18. In his celebrated work, Thomason essentially proved that a graph distributed as $G(n, p)$ is $(p, O(1))$-pseudorandom with high probability 1 On the other hand, there is no infinite sequence of $(p, o(1))$-pseudorandom graphs.

The following lemma can be easily verified by combining a Chernoff type bound with the union bound.

Lemma 1.1. For every constant $c>0$ there is a constant $C>0$ such that for $p \geq \frac{C}{n}, a$ random directed graph $G \in D(n, p)$ is $(p, c)$-pseudorandom with high probability.

Our results in this work will hold for every $(p, r)$-pseudorandom graph with $p \geq \frac{C}{n}$ for some sufficiently large constant $C$, and every $r \leq \mu \sqrt{p n}$ for some small constant $\mu>0$ that will be chosen later. By Lemma 1.1, a random directed graph distributed according to $D(n, p)$ with $p \geq \frac{C}{n}$ has this property with high probability.

We show here that the directed case is both similar and different from the undirected case. In fact, since we reduce here the directed case to the global resilience problem of the undirected case, we can use ideas from Dellamonica et al. [10] in order to get our bounds on the resilience for directed graphs. On the other hand, many of the techniques that were used for the undirected case cannot be applied in the directed case. Also, the range of parameters relevant to us is rather different, since in particular the result of Dellamonica et al. [10] shows that the removal of any 0.99-fraction of the edges of a (pseudo)random undirected graph still leaves a cycle of linear size. For the directed case it is easy to see that one can always remove half of the edges of any directed graph and get an acyclic directed graph, and hence a graph with no cycles at all.

\footnotetext{
${ }^{1}$ Here a sequence of events $A_{n}, n \geq 1$ is said to occur with high probability if $\lim _{n \rightarrow \infty} \mathbb{P}\left[A_{n}\right]=1$.
} 


\subsection{Our results}

Woodall [19] studied the minimal number of edges that guarantees the existence of a long cycle. In our terminology, he studied the global resilience of the complete graph $K_{n}$ with respect to the property of having a cycle of length at least $\ell$. He proved the following.

Theorem 1 (Woodall [19]). Let $3 \leq \ell \leq n$. Every graph $G$ on $n$ vertices satisfying

$$
e(G) \geq\left\lceil\frac{n-1}{\ell-2}\right\rceil \cdot\left(\begin{array}{c}
\ell-1 \\
2
\end{array}\right)+\left(\begin{array}{c}
r+1 \\
2
\end{array}\right)+1,
$$

where $r=(n-1) \bmod (\ell-2)$, has a cycle of length at least $\ell$.

It is easy to verify that Woodall's bound is best possible. Indeed, take a graph formed by $\left\lceil\frac{n-1}{\ell-2}\right\rceil$ disjoint cliques of size $\ell-2$, a single smaller clique of size $r$ and a vertex that is connected to every other vertex in the graph. Clearly, the length of the longest cycle in this graph is at most $\ell-1$.

The work of Dellamonica et al. [10] can be viewed as a generalization of Woodall's work from the case of $K_{n}$ to the case of general pseudorandom graphs. In order to cite their result and also for future reference in our paper the following function is defined.

Definition 2. For a given $0 \leq \alpha<1$, define

$$
w(\alpha)=1-(1-\alpha)\left\lfloor(1-\alpha)^{-1}\right\rfloor .
$$

It is easy to verify that we have $w(0)=0$ and $\lim _{x \nearrow_{1}} w(x)=0$. The following asymptotic version of Woodall's result is proved in [10] and is easier to work with.

Theorem 3 ( [10]). Let $\alpha>0$. For every $\beta>0$ there is $n_{0}$ such that for every graph $G$ on $n>n_{0}$ vertices satisfying

$$
|E(G)| \geq\left(\begin{array}{l}
n \\
2
\end{array}\right) \cdot(1-(1-w(\alpha))(\alpha+w(\alpha))+\beta)
$$

has a cycle of length at least $(1-\alpha) \cdot n$.

Dellamonica et al. proved in [10 that Theorem 3 can be extended to (sparse) pseudorandom graphs; more specifically they proved that any subgraph $G^{\prime}=\left(V, E^{\prime}\right)$ of a pseudorandom graph $G=(V, E)$ with $\left|E^{\prime}\right| \geq(1-(1-w(\alpha))(\alpha+w(\alpha))+o(1))|E|$ edges has a cycle of length at least $(1-\alpha) \cdot|V|$. Here we obtain a directed analogue of their result.

We can now state our main theorem.

Theorem 4. Fix $0<\gamma<\frac{1}{2}$ and let $G=(V, E)$ be a $(p, r)$-pseudorandom directed graph on $n$ vertices, where $r \leq \mu \sqrt{n p}$ and $\mu(\gamma)>0$ is a sufficiently small constant that depends only on $\gamma$ and $n$ is sufficiently large. Let $G^{\prime}$ be the subgraph of $G$ with at least $\left(\frac{1}{2}+\gamma\right)|E|$ edges. Then $G^{\prime}$ contains a directed cycle of length at least $(1-\alpha-o(1)) \cdot n$, where $\alpha$ satisfies

$$
2 \gamma=1-(1-w(\alpha))(\alpha+w(\alpha)) .
$$


Observe crucially that every directed graph $G=(V, E)$ contains an acyclic subgraph $G^{\prime}$ with at least $|E| / 2$ edges. Indeed, fix a permutation $\sigma: V \rightarrow V$, and let $G_{1}$ be the subgraph with all edges $x y$ such that $\sigma(x)>\sigma(y)$, and $G_{2}$ be the subgraph with all edges $x y$ such that $\sigma(x)<\sigma(y)$. Then both $G_{1}$ and $G_{2}$ are acyclic, and at least one of them contains at least half of the edges of $G$.

Theorem 4 yields the following two immediate corollaries.

Corollary 5. For every $\gamma>0$ there is a constant $c_{1}(\gamma)>0$ such that the following holds. Let $G$ be a $(p, r)$-pseudorandom graph on $n$ vertices, $r \leq \mu \sqrt{n p}$ where $\mu(\gamma)>0$ is some sufficiently small constant that depends only on $\gamma$ and $n$ is sufficiently large. Let $G^{\prime}$ be a subgraph of $G$ with at least $(1 / 2+\gamma)|E(G)|$ edges. Then $G^{\prime}$ contains a directed cycle of length at least $c_{1} n$.

In words, the above corollary guarantees that the deletion of less than half of the edges of a pseudorandom digraph leaves a cycle of linear length.

Corollary 6. There exists a function $c_{2}(\epsilon)$ with $\lim _{\epsilon \rightarrow 0} c_{2}(\epsilon)=0$ such that the following holds. Let $G$ be a (p,r)-pseudorandom graph on $n$ vertices, $r \leq \mu \sqrt{n p}$ where $\mu(\gamma)>0$ is some sufficiently small constant that depends only on $\gamma$ and $n$ is sufficiently large. Let $G^{\prime}$ be a subgraph of $G$ with at least $(1-\varepsilon)|E(G)|$ edges. Then $G^{\prime}$ contains a directed cycle of length at least $\left(1-c_{2}\right) \cdot n$.

Here, we have that deleting a negligible fraction of the edges of a pseudorandom digraph leaves a cycle of length close to $n$.

Finally, we prove the following matching lower bound.

Proposition 7. Fix $0<\gamma<\frac{1}{2}$ and let $G$ be a (p,r)-pseudorandom directed graph on $n$ vertices, where $r=O(\sqrt{n p})$ and $p n \rightarrow \infty$. There is a subgraph $G^{\prime}$ with $\left(\frac{1}{2}+\gamma\right)|E|$ edges that does not contain any directed cycle of length $(1-\alpha+o(1)) \cdot n$, where $\alpha$ satisfies

$$
2 \gamma=1-(1-w(\alpha))(\alpha+w(\alpha))
$$

Our Tools. One of the main tools we use in this work is a sparse directed variant of Szemerédi's regularity lemma (Lemma 2.1), that was stated in [11]. This allows us to partition our graph to a constant number of regular pairs, and essentially to reduce the problem to finding almost spanning paths in regular pairs.

To this end, we use a simple yet powerful lemma that finds almost spanning paths in expanding graphs (Lemma 2.3). In our case, a regular pair is a bipartite expander is both directions. The approach is based on ideas from [9, 2, 4].

The rest of the paper is organized as follows. In Section 2 we state the sparse directed regularity lemma, and prove that regular pairs have an almost spanning directed path. In Section 3, we reduce the resilience problem in directed graphs to undirected graphs, and then apply ideas from [10]. In Section 4 we show that our results are essentially tight. 
Throughout the proofs we assume that the order of $G$, denoted by $n$, is large enough. We do not try to optimize constants and omit floor and ceil signs whenever these are not crucial.

\section{The regularity lemma for sparse directed graphs and long paths in regular pairs}

\subsection{The regularity lemma}

In this section we follow [11] and state a regularity lemma for sparse directed graphs. We first provide some notation.

Given a directed graph $G=(V, E)$, for any pair of disjoint sets of vertices $U, W$, we let $E_{G}(U, W)$ be the set of edges directed from $U$ to $W$, and let $e_{G}(U, W)=\left|E_{G}(U, W)\right|$. We say that $G$ is $(\delta, D, p)$-bounded if for any two sets $U, W$ such that $|U|,|W| \geq \delta|V|$ we have

$$
e_{G}(U, W) \leq D p|U||W|
$$

The edge density from a set $U$ to a set $W$ is defined by $\frac{e_{G}(U, W)}{|U||W|}$. We say that two sets $U$ and $W$ span a bipartite directed graph of bi-density $p$ if it has edge density at least $p$ in both directions. Also define the directed $p$-density from $U$ to $W$ by

$$
d_{G, p}(U, W)=\frac{e_{G}(U, W)}{p|U||W|} .
$$

We omit the index graph $G$ and write $d_{p}(U, W)$ whenever the base graph is clear from the context.

For $0<\delta \leq 1$, a pair $(U, W)$ is $(\delta, p)$-regular in a digraph $G$ if for every $U^{\prime} \subseteq U$ and $W^{\prime} \subseteq W$ such that $\left|U^{\prime}\right| \geq \delta|U|$ and $\left|W^{\prime}\right| \geq \delta|W|$ we have both

$$
\left|d_{G, p}(U, W)-d_{G, p}\left(U^{\prime}, W^{\prime}\right)\right|<\delta,
$$

and

$$
\left|d_{G, p}(W, U)-d_{G, p}\left(W^{\prime}, U^{\prime}\right)\right|<\delta .
$$

A partition $\mathcal{P}=\left\{V_{0}, V_{1}, \ldots, V_{k}\right\}$ of $V$ is $(\delta, k, p)$-regular if the following properties hold.

(i). $\left|V_{0}\right| \leq \delta|V|$.

(ii). $\left|V_{i}\right|=\left|V_{j}\right|$ for all $1 \leq i<j \leq k$.

(iii). At least $(1-\delta)\left(\begin{array}{l}k \\ 2\end{array}\right)$ of the pairs $\left(V_{i}, V_{j}\right), 1 \leq i<j \leq k$, are $(\delta, p)$-regular.

We will use the following variant of Szemerédi's regularity lemma, that is stated in [11], and its proof follows lines similar to the proof of the regularity lemma for sparse graphs, proved independently by Kohayakawa and by Rödl (see, e.g., [14]). In [11] the lemma is stated for oriented graphs (where no anti parallel edges are allowed), yet the result can be easily adjusted to our case, where anti parallel edges are allowed. 
Lemma 2.1 (Lemma 3 in [11]). For any real number $\delta>0$, an integer $k_{0} \geq 1$ and real number $D>1$, there exist constants $\eta=\eta\left(\delta, k_{0}, D\right)$ and $K=K\left(\delta, k_{0}, D\right) \geq k_{0}$ such that for any $0<p(n) \leq 1$, any $(\eta, D, p)$-bounded directed graph $G$ admits a $(\delta, k, p)$-regular partition for some $k_{0} \leq m \leq K$.

\subsection{Every regular pair contains a long path}

We next prove that every regular pair of positive bi-density contains an almost spanning path. To this end, we first show a trivial expansion property of regular pairs, and then apply this property to prove the desired result.

Claim 2.2. Let $(U, W)$ be a $(\delta, p)$-regular pair for $|U|=|W|$ with bi-density at least $2 \delta p$, where $p>0$. Then for every two sets $U^{\prime} \subseteq U$ and $W^{\prime} \subseteq W$ such that $\left|U^{\prime}\right| \geq \delta|U|$ and $\left|W^{\prime}\right| \geq \delta|W|$ there is a directed edge from $U^{\prime}$ to $W^{\prime}$.

Proof. By regularity we have

$$
e_{G}\left(U^{\prime}, W^{\prime}\right) \geq\left(d_{p}(U, W)-\delta\right) p\left|U^{\prime}\right|\left|W^{\prime}\right| \geq(2 \delta-\delta) p\left|U^{\prime}\right|\left|W^{\prime}\right|=\delta p\left|U^{\prime}\right|\left|W^{\prime}\right|>0 .
$$

The claim follows.

We next show that a bipartite directed graph with a simple expansion property contains a long directed path. The proof follows ideas from [9, 2, 4].

Lemma 2.3. Let $H=\left(V_{1}, V_{2}, E\right),\left|V_{1}\right|=\left|V_{2}\right|=t$, be a directed bipartite graph that satisfies the following property: for every two sets $A \subseteq V_{1}, B \subseteq V_{2}$ of size $k$, there is at least one edge from $A$ to $B$ and there is at least one edge from $B$ to $A$. Then $H$ contains a directed path of length $2 t-4 k+3$.

Proof. Recall that the DFS (Depth First Search) is a graph search algorithm that visits all the vertices of a (directed or undirected) graph $G$ as follows. It maintains three sets of vertices, letting $S$ be the set of vertices which we have completed exploring, $T$ be the set of unvisited vertices, and $U=V(G) \backslash(S \cup T)$, where the vertices of $U$ are kept in a stack (a last in, first out data structure). It is also assumed that some order $\sigma$ on the vertices of $G$ is fixed, and the algorithm prioritizes vertices according to $\sigma$. The DFS starts with $S=\emptyset, U=\left\{\sigma_{1}\right\}$ and $T=V(G) \backslash\left\{\sigma_{1}\right\}$.

While there is a vertex in $V(G) \backslash S$, if $U$ is non-empty, let $v$ be the last vertex that was added to $U$. If $v$ has an out-neighbor $u \in T$, the algorithm inserts $u$ to $U$. If $v$ does not have an out-neighbor in $T$ then $v$ is popped out from $U$ and is moved to $S$. If $U$ is empty, the algorithm chooses an arbitrary vertex from $T$ and pushes it to $U$.

We now proceed to the proof of the lemma. We execute the DFS algorithm for an arbtrary chosen order $\sigma$ on the vertices of the graph. We let again $S, T, U$ be three sets of vertices as defined above. At the beginning of the algorithm, all the vertices are in $T$, and at each step a single vertex either moves from $T$ to $U$ or from $U$ to $S$. At the end of the algorithm, all the vertices are in $S$. 
Consider the point during the execution of the algorithm when $|S|=|T|$. Observe crucially that all the vertices in $U$ form a directed path, and we have $\left\|U \cap V_{1}|-| U \cap V_{2}\right\| \leq 1$. Since $|U|=2 t-|S|-|T|=2 t-2|S|$ is even, we have in fact

$$
\left|U \cap V_{1}\right|=\left|U \cap V_{2}\right| .
$$

We get that

$$
|S|=\left|S \cap V_{1}\right|+\left|S \cap V_{2}\right|=\left|T \cap V_{1}\right|+\left|T \cap V_{2}\right|=|T|,
$$

and

$$
\left|V_{1} \backslash U\right|=\left|S \cap V_{1}\right|+\left|T \cap V_{1}\right|=\left|S \cap V_{2}\right|+\left|T \cap V_{2}\right|=\left|V_{2} \backslash U\right| .
$$

Hence, we get both

$$
\left|S \cap V_{2}\right|=\left|T \cap V_{1}\right|,
$$

and

$$
\left|S \cap V_{1}\right|=\left|T \cap V_{2}\right| .
$$

Assume without loss of generality that

$$
\left|S \cap V_{1}\right| \geq|S| / 2 \geq\left|S \cap V_{2}\right| .
$$

Then $\left|S \cap V_{1}\right| \geq t / 2-|U| / 4$ and therefore $\left|T \cap V_{2}\right| \geq t / 2-|U| / 4$. Observe crucially that there are no edges from $S$ to $T$. By the assumption of the lemma we conclude $\left|S \cap V_{1}\right|,\left|T \cap V_{2}\right| \leq k-1$ and therefore we get $t / 2-|U| / 4 \leq k-1$ and hence $|U| \geq 2 t-4 k+4$. Thus $H$ contains a directed path $|U|$ of length $2 t-4 k+3$, as desired.

We therefore have the following corollary.

Corollary 2.4. Let $(U, W)$ be a $(\delta, p)$-regular pair with bi-density at least $2 \delta p$ and $|U|=$ $|W|=t, p>0$. Then the bipartite directed graph between $U$ and $W$ contains a directed path of length $(1-2 \delta) \cdot 2 t+2$ that starts at $U$.

Proof. By Claim 2.2, there is an edge in each direction between every two sets of size $\delta t$ in $U$ and $W$. Therefore Lemma 2.3 implies the existence of a directed path of length $(1-2 \delta) 2 t+3$. Note that if the first vertex in the path is from $W$ we may remove it, thus getting a directed path of length at least $(1-2 \delta) 2 t+2$ that starts at $U$.

\section{Proof of Theorem 4}

In this section we prove our main result. Given a constant $\gamma>0$, we essentially want to prove that every subgraph with $(1 / 2+\gamma)$-fraction of the edges of a pseudorandom directed graph contains a long directed cycle. Let $\delta=\delta(\gamma)$ to be fixed later, $K=K(\delta, 1 / \delta, 1+\delta)$ and $\eta=\eta(\delta, 1 / \delta, 1+\delta)$ be the constants defined by the regularity lemma (Lemma 2.1). 
Let $G=(V, E)$ be a $(p, r)$-pseudorandom directed graph with $r \leq \mu \sqrt{n p}$. For $\mu \leq$ $\delta \cdot \min \{\eta, 1 / K\}$ we have

$$
r \leq \delta \sqrt{n p} \cdot \min \{\eta, 1 / K\} .
$$

Let $A, B$ be two sets of vertices of size $\eta n$ in $G$. Observe that

$$
r \sqrt{p n|A||B|} \leq \delta \sqrt{n p} \eta \sqrt{p \eta^{2} n^{3}}=\delta \eta^{2} n^{2} p \leq \delta \eta^{2} n^{2}=\delta|A||B| .
$$

Therefore, we get that $G$ is $(\eta, 1+\delta, p)$-bounded.

Given a subgraph $G^{\prime}=\left(V, E^{\prime}\right)$ of $G$ that contains $(1 / 2+\gamma)|E|$ edges, our goal is to show that $G^{\prime}$ contains a long directed cycle.

Clearly, $G^{\prime}$ is $(\eta, 1+\delta, p)$-bounded as well, and hence we can apply the sparse directed regularity lemma (Lemma 2.1) to $G^{\prime}$ and get a partition of $V$ to clusters $V_{0}, V_{1}, \ldots, V_{m}$, where $1 / \delta \leq m \leq K,\left|V_{0}\right| \leq \delta n,\left|V_{1}\right|=\left|V_{2}\right|=\cdots=\left|V_{m}\right|=t$ and all but at most $\delta$-fraction of the pairs $\left(V_{i}, V_{j}\right)$ are $(\delta, p)$-regular. Note that $\frac{n(1-\delta)}{m} \leq t \leq \frac{n}{m}$.

We next define an undirected auxiliary graph $H$ on the clusters $V_{1}, \ldots, V_{m}$. With a slight abuse of notation, we denote the vertices of $H$ by $V_{1}, V_{2}, \ldots, V_{m}$. Two vertices $V_{i}$ and $V_{j}$ are connected if the pair $\left(V_{i}, V_{j}\right)$ is $(\delta, p)$-regular and has bi-density at least $2 \delta p$.

Since $G$ is $(p, r)$-pseudorandom and $r \leq \frac{\delta \cdot \sqrt{n p}}{K} \leq \frac{\delta \cdot \sqrt{n p}}{m}$, we get that the edge density of every pair $\left(V_{i}, V_{j}\right)$ in $G$ is at least $1-\delta$ and at most $1+\delta$.

Observe that if $V_{i}$ and $V_{j}$ are not connected by an edge in $H$, one of the following must happen.

- The pair $\left(V_{i}, V_{j}\right)$ is not regular.

- Either $\left|E_{G^{\prime}}\left(V_{i}, V_{j}\right)\right|<2 \delta p\left|V_{i}\right|\left|V_{j}\right|$ or $\left|E_{G^{\prime}}\left(V_{j}, V_{i}\right)\right|<2 \delta p\left|V_{i}\right|\left|V_{j}\right|$. In other words, at least $(1-3 \delta) p\left|V_{i}\right|\left|V_{j}\right|$ of the edges from $V_{i}$ to $V_{j}$ or from $V_{j}$ to $V_{i}$ in $G$ are not in $G^{\prime}$.

The number of non-regular pairs is at most $\delta\left(\begin{array}{c}m \\ 2\end{array}\right)$. Since in every pair with bi-density less than $2 \delta p$ at least $(1-3 \delta) p t^{2}$ edges were lost when moving from $G$ to $G^{\prime}$, we get that the number of pairs in $G^{\prime}$ with edge density less than $2 \delta p$ is bounded by

$$
\frac{(1 / 2-\gamma) p n^{2}}{(1-3 \delta) p t^{2}} \leq \frac{(1 / 2-\gamma) p n^{2}}{(1-3 \delta) p\left(\frac{n(1-\delta)}{m}\right)^{2}}=\frac{1 / 2-\gamma}{(1-3 \delta)(1-\delta)^{2}} \cdot m^{2} .
$$

Observe that by our choice of parameters for the regularity lemma we have $m \geq \frac{1}{\delta}$, and hence we have

$$
m^{2} \leq 2\left(\begin{array}{c}
m \\
2
\end{array}\right)(1+2 / m) \leq 2\left(\begin{array}{c}
m \\
2
\end{array}\right)(1+2 \delta) .
$$

We conclude that the fraction of non-edges in $H$ is bounded by

$$
\delta+\frac{(1-2 \gamma)(1+2 \delta)}{(1-3 \delta)(1-\delta)^{2}}
$$

By taking $\delta \ll \gamma$, we get that the fraction of edges in $H$ is at least $2 \gamma(1-o(1))$, where the $o(1)$ term depends only on $\delta$ and can be made arbitrary small. 
Let $\alpha$ be the minimal solution of the equation

$$
2 \gamma=1-(1-w(\alpha))(\alpha+w(\alpha)),
$$

where $w$ is the function defined in Subsection 1.2. By Theorem 3, we get that $H$ contains an undirected cycle of length $(1-\alpha-o(1)) m$.

We complete the proof by showing how to construct a long cycle in $G^{\prime}$ given a long cycle in $H$. We start with the case that $H$ contains a long cycle of even length, and later show how to modify the proof in the case of odd length.

Let $V_{i_{1}}, V_{i_{2}}, \ldots V_{i_{2 b}}$ be a cycle of length $(1-\alpha+o(1)) m$ in $H$. Note that for every $1 \leq q \leq 2 b$, the pair $\left(V_{i_{q}}, V_{i_{q+1}}\right)$ (where we identify $V_{i_{2 b+1}}$ with $\left.V_{i_{1}}\right)$ is $(\delta, p)$-regular and has edge bi- density at least $2 \delta p$. Therefore, by Corollary 2.4, for every $1 \leq q \leq b$, there is a directed path $P_{q}$ of length at least $(1-2 \delta) \cdot 2 t$ that alternates between $V_{i_{2 q-1}}$ and $V_{i_{2 q}}$. For every $1 \leq q \leq b$, let $P_{q}^{R}=P_{q} \cap V_{i_{2 q}}$ and let $P_{q}^{L}=P_{q} \cap V_{i_{2 q-1}}$.

Observe that for every $1 \leq q \leq b$, by Claim 2.2 there is an edge from the last $\delta t$ vertices of $P_{q}^{R}$ to the first $\delta t$ vertices if $P_{q+1}^{L}$ (here we identify $P_{b+1}^{L}$ with $P_{1}^{L}$ ). Thus we can paste every two consecutive paths together, losing at most $2 \delta t$ vertices from each path. We conclude that we can paste all the paths together to get a directed cycle of length

$$
b \cdot(1-2 \delta-\delta) \cdot 2 t=(1-\alpha-o(1)) m t=(1-\alpha-o(1))\left|V\left(G^{\prime}\right)\right|,
$$

as claimed.

Finally, suppose there is an odd cycle of length $(1-\alpha+o(1)) m$ in $H$, whose vertices are by $V_{i_{1}}, V_{i_{2}}, \ldots V_{i_{2 b+1}}$. For every $1 \leq q \leq b$, let $P_{q}$ be a path of length at least $(1-2 \delta) \cdot 2 t$ that alternates between $V_{i_{2 q-1}}$ and $V_{i_{2 q}}$. Moreover, let

$$
V_{i_{2 b+1}}^{\prime}=\left\{v \in V_{i_{2 b+1}}: \forall u \in P_{b}^{R},(u, v) \notin E\right\},
$$

and

$$
V_{i_{2 b+1}}^{\prime \prime}=\left\{v \in V_{i_{2 b+1}}: \forall u \in P_{1}^{L},(v, u) \notin E\right\} .
$$

By Claim [2.2, we have $\left|V_{i_{2 b+1}}^{\prime}\right|,\left|V_{i_{2 b+1}}^{\prime \prime}\right| \leq \delta t$, and therefore for all but $2 \delta t$ of the vertices in $V_{i_{2 b+1}}$ have an edge from the last $2 \delta t$ vertices in $P_{b}$ and an edge to the first $2 \delta t$ first vertices in $P_{1}$. Since $t>2 \delta t$ we conclude that we can connect $P_{b}$ and $P_{1}$ through a vertex in $V_{i_{2 b+1}}$, thus getting a path of length

$$
b \cdot(1-2 \delta-\delta) 2 t=(1-\alpha+o(1))\left|V\left(G^{\prime}\right)\right| .
$$

Theorem 4 follows.

\section{Lower bounds}

Let $G=(V, E)$ be a directed graph. Recall that by fixing a permutation $\sigma$ on the vertices, we can partition the edges of $G$ to two acyclic sets as follows. The first set contains all 
directed edges $x y$ where $\sigma(x)>\sigma(y)$, and the second set contains all directed edges $x y$ where $\sigma(y)>\sigma(x)$. Therefore, the global resilience of every directed graph with respect to the property of having directed cycles is at most $1 / 2$. Here we extend this idea and show that our main result is asymptotically tight.

Proof of Proposition 7. We show that there is a subgraph $G^{\prime}$ with $(1 / 2+\gamma)$-fraction of the edges, whose longest directed cycle is of length at most $(1-\alpha+o(1)) n$. Our approach follows [10].

Recall that $G$ is $(p, r)$-pseudorandom with $r \leq \mu \sqrt{n p}$ and $p n \rightarrow \infty$. We first claim that for every two disjoint sets $A, B$ of size $\Omega(n)$, the number of edges from $A$ to $B$ is $p|A||B|(1+o(1))$. Indeed, let $|A|=a n$ and $|B|=b n$, and suppose that $a<b$. Let $B^{\prime}$ be a random subset of $B$ of size an, then by linearity of expectation the number of edges between $A$ and $B^{\prime}$ is $E(A, B) \cdot \frac{\left|B^{\prime}\right|}{B}$. Therefore, if the number of edges between $A$ and $B$ is smaller than (respectively, larger than) from $p|A||B|(1+o(1))$ then there is a choice of a set $B^{\prime}$ such that $A$ and $B^{\prime}$ contradicts the $(p, r)$-pseudorandomness of $G$.

We partition the vertices of $G$ into $k$ classes $V_{1}, V_{2}, \ldots, V_{k}$ of size $(1-\alpha) n$ each, and one additional class $V_{k+1}$ of size $w(\alpha) n \leq(1-\alpha) n$. Let $G^{\prime}$ be the subgraph with all edges from $V_{i}$ to $V_{j}$, for $1 \leq i<j \leq k+1$, and all the edges within each class $V_{i}$. Clearly, $G^{\prime}$ does not contain a cycle longer than $(1-\alpha) n$, since a directed path leaving a certain $V_{i}$ cannot return there. Therefore, we can conclude the proof by showing that the number of edges in $G^{\prime}$ is as claimed.

Indeed, since $G$ is $(p, r)$-pseudorandom and each $V_{i}$ is of size $\Omega(n)$, we get that for every $1 \leq i<j \leq k$, the number of edges from $V_{j}$ to $V_{i}$ is $(1-\alpha)^{2} \cdot p n^{2}(1+o(1))$. The number of edges from $V_{k+1}$ to $\cup_{i \leq k} V_{i}$ in $G$ is $k(1-\alpha) w(\alpha) \cdot p n^{2}(1+o(1))$. Recalling that by definition of $w(x)$ we have $(1-\alpha) k=1-w(\alpha)$, we get that the number of edges we deleted from $G$ to get $G^{\prime}$ is

$$
\begin{aligned}
& \left(\left(\begin{array}{l}
k \\
2
\end{array}\right)(1-\alpha)^{2}+k(1-\alpha) w(\alpha)\right) \cdot p n^{2}(1+o(1)) \\
& =(1-\alpha) k((1-\alpha)(k-1)+2 w(\alpha))(1+o(1)) \frac{p n^{2}}{2} \\
& =(1-w(\alpha))(\alpha-w(\alpha)+2 w(\alpha)) \cdot \frac{p n^{2}(1+o(1))}{2} \\
& =(1-w(\alpha))(\alpha+w(\alpha))+o(1)) \frac{p n^{2}}{2} .
\end{aligned}
$$

Note that the number of edges in $G$ is $p n^{2}(1+o(1))$. Let the number of edges in $G^{\prime}$ be $(1 / 2+\gamma) \cdot p n^{2}$. Then the number of edges we deleted satsifies

$$
\left.(1+o(1))(1 / 2-\gamma) p n^{2}=(1-w(\alpha))(\alpha+w(\alpha))+o(1)\right) \frac{p n^{2}}{2},
$$

and therefore $\gamma$ satisfies

$$
2 \gamma=1-(1-w(\alpha))(\alpha+w(\alpha))+o(1)),
$$


as claimed. The statement follows.

\section{Concluding remarks}

We studied the global resilience of pseudorandom directed graphs with respect to the property of having a long directed cycle. We gave matching lower and upper bounds, and our proof essentially reduced our problem to case of undirected graphs.

A variety of questions regarding the resilience of directed graphs can be asked. A few, somewhat arbitrary examples are the problem of local resilience with respect to having a long directed cycle, the resilience with respect to the property of having some fixed directed graph. Another interesting problem is the resilience with respect to Hamilitonicity, which in the dense case is settled in [12].

In this work we considered subgraphs with $(1 / 2+\gamma)$-fraction of the edges, and observed that every directed graph contains an acyclic subgraph with $1 / 2$-fraction of the edges. In [4], the authors proved that every two-coloring of the edges of a pseudorandom digraph contains a relatively long monochromatic path. That is, instead of proving that a large subgraph has a certain property, it is proved that every partition of the edges of the graph has a certain property. It will be interesting to give such results for other properties of directed graphs.

\section{References}

[1] N. Alon and B. Sudakov, Increasing the chromatic number of a random graph, submitted.

[2] J. Bang-Jensen and S. Brandt, Expansion and Hamiltonicity in Digraphs, manuscript.

[3] J. Balogh, B. Csaba and W. Samotij, Local resilience of almost spanning trees in random graphs, Random Struct. Algorithms, to appear.

[4] I. Ben-Eliezer, M. Krivelevich and B. Sudakov, The size Ramsey number of a directed path, submitted.

[5] S. Ben-Shimon, M. Krivelevich and B. Sudakov, Local resilience and Hamiltonicity Maker-Breaker games in random regular graphs, submitted.

[6] J. C. Bermond and C. Thomassen, Cycles in digraphs - A survey, Journal of Graph Theory, 5 (1981), 1-43, .

[7] B. Bollobás, Extremal graph theory, Dover 2004.

[8] J. Böttcher, Y. Kohayakawa, and A. Taraz, Almost spanning subgraphs of random graphs after adversarial edge removal, arXiv:1003.0890v1. 
[9] S. Brandt, H. Broersma, R. Diestel and M. Kriesell, Global connectivity and expansion: long cycles in $f$-connected graphs, Combinatorica 26 (2006), 17-36.

[10] D. Dellamonica Jr. , Y. Kohayakawa, M. Marciniszyn and A. Steger, On the resilience of long cycles in random graphs, Electronic Journal of Combinatorics 15, 2008, R32.

[11] J. Donadelli and Y. Kohayakawa, A density result for random sparse oriented graphs and its relation to a conjecture of Woodall, Electronic Journal of Combinatorics 9 (2002), no. 1, Research paper 45.

[12] D. Hefetz, A. Steger and B. Sudakov, in perpartion.

[13] H. Huang, C. Lee and B. Sudakov, Bandwidth theorem for sparse graphs, submitted.

[14] Y. Kohayakawa, Szemerédi's regularity lemma for sparse graphs, Foundations of Computational Mathematics (Berlin, Heidelberg) (F. Cucker and M. Shub, eds.), Springer-Verlag, January 1997, pp. 216-230.

[15] M. Krivelevich, C. Lee and B. Sudakov, Resilient pancyclicity of random and pseudorandom graphs, SIAM Journal on Discrete Mathematics, 24(1), 1-16, 2010.

[16] M. Lewin, On maximal circuits in directed graphs, Journal of Combinatorial Theory, Series B, 18(2), 1975, 175-179.

[17] B. Sudakov and V. Vu, Local resilience of graphs, Random Structures and Algorithms 33 (2008), 409-433.

[18] A. Thomason, Pseudo-random graphs, 2nd International Seminar on Random Graphs and Probabilistic Methods in Combinatorics 144 (1985), 307-331.

[19] D. R. Woodall, Sufficient conditions for circuits in graphs, Proc. London Math. Soc. (3), 24:739-755, 1972. 